\title{
On the exponential decay of the correlation functions for KMO-Langevin equations
}

\author{
By Yasunori OKabe and Akihiko Inoue \\ (Received November 8, 1990) \\ (Revised December 14, 1990)
}

\section{§1. Introduction}

We consider the first continuous KMO-Langevin equation

$$
\dot{\mathbf{X}}=-\beta_{1} \mathbf{X}-\lim _{\varepsilon \downarrow 0} \gamma_{1, \varepsilon} * \dot{\mathbf{X}}+\alpha_{1} \dot{\mathbf{B}}
$$

and the second continuous KMO-Langevin equation

$$
\dot{\mathbf{X}}=-\beta_{2} \mathbf{X}-\lim _{\varepsilon \downarrow 0} \gamma_{2, \varepsilon} * \dot{\mathbf{X}}+\alpha_{2} \mathbf{I} .
$$

Here, for $j=1,2, \alpha_{j}$ and $\beta_{j}$ are positive constants and for $t \in \mathbf{R}$ and $\varepsilon>0$,

$$
\gamma_{j, \varepsilon}(t)=\chi_{(0, \infty)}(t) \int_{\varepsilon}^{\infty} e^{-t \lambda} \rho_{j}(d \lambda)
$$

with a Borel measure $\rho_{j}$ on $[0, \infty)$ satisfying

$$
\rho_{j}(\{0\})=0, \quad \int_{0}^{\infty}(\lambda+1)^{-1} \rho_{j}(d \lambda)<\infty .
$$

$\dot{\mathbf{B}}$ is a Gaussian white noise and $\mathbf{I}$ is a stationary Gaussian random tempered distribution named the Kubo noise associated with the solution of (1.2) (see [3]).

The equation (1.1) (resp. (1.2)) has a unique solution $X_{1}=\left(X_{1}(t) ; t \in \mathbf{R}\right)$ (resp. $\mathbf{X}_{2}=\left(X_{2}(t) ; t \in \mathbf{R}\right)$ ) which is a real stationary Gaussian process with mean zero and covariance function $R_{1}$ (resp. $R_{2}$ ) of the form

$$
R_{j}(t)=\int_{0}^{\infty} e^{-|t| \lambda} \sigma_{j}(d \lambda) \quad(t \in \mathbf{R}) .
$$

Here $\sigma_{j}$ is a non-zero bounded Borel measure on $[0, \infty)$ satisfying

$$
\begin{array}{ll}
\sigma_{1}(\{0\})=0, & \int_{0}^{\infty}\left(\lambda+\lambda^{-1}\right) \sigma_{1}(d \lambda)<\infty \\
\sigma_{2}(\{0\})=0, & \int_{0}^{\infty} \lambda^{-1} \sigma_{2}(d \lambda)<\infty
\end{array}
$$


In fact, $\sigma_{j}$ is given by $\sigma_{j}=L_{j}{ }^{-1}\left(\left(\alpha_{j}, \beta_{j}, \rho_{j}\right)\right)$, where $L_{1}$ and $L_{2}$ are defined by (3.2) and (2.3) respectively. The property (1.5) is called the reflection positivity. Conversely, it is known that any real stationary Gaussian process $\mathbf{X}$ with the reflection positivity becomes a solution of both (1.1) and (1.2) for some adequate triples $\left(\alpha_{1}, \beta_{1}, \gamma_{1}\right)$ and $\left(\alpha_{2}, \beta_{2}, \gamma_{2}\right)$, respectively. For the details, see [3].

We put for $j=1,2$ and $t \in \mathbf{R}$,

$$
\gamma_{j}(t)=\chi_{(0, \infty)}(t) \int_{0}^{\infty} e^{-t \lambda} \rho_{j}(d \lambda)
$$

In [7] and [1], the long time tail behavior of $R_{1}$ (resp. $R_{2}$ ) was characterized by $\gamma_{1}$ (resp. $\gamma_{2}$ ) as follows: when $t \rightarrow \infty$,

$$
\begin{aligned}
& \gamma_{1}(t) \sim t^{-p} L(t) \Longleftrightarrow R_{1}(t) \sim \frac{\alpha_{1}^{2} p}{\beta_{1}{ }^{3}} t^{-(1+p)} L(t), \\
& \gamma_{2}(t) \sim t^{-p} L(t) \Longleftrightarrow R_{2}(t) \sim \frac{\sqrt{2 \pi} \alpha_{2} p}{\beta_{2}{ }^{2}} t^{-(1+p)} L(t),
\end{aligned}
$$

where $0<p<\infty$ and $L$ is a slowly varying function at infinity.

In this paper, we show that the exponential decay of $R_{j}$ can be also characterized by the decay of $\gamma_{j}$. We put

$$
\lambda_{0}\left(\sigma_{j}\right)=\inf \left\{\lambda ; \lambda \in \operatorname{supp} \sigma_{j}\right\}, \quad \lambda_{0}\left(\rho_{j}\right)=\inf \left\{\lambda ; \lambda \in \operatorname{supp} \rho_{j}\right\}
$$

Then we see that

$$
\lim _{t \rightarrow \infty} \frac{\log R_{j}(t)}{t}=-\lambda_{0}\left(\sigma_{j}\right), \quad \lim _{t \rightarrow \infty} \frac{\log \gamma_{j}(t)}{t}=-\lambda_{0}\left(\rho_{j}\right)
$$

(Proposition 3.1). Using (1.12), we show the following theorem in $\S 3$.

THEOREM 1.1. Let $j=1$ or 2 . Then the following (i) and (ii) are equivalent:

$$
\begin{aligned}
& \lim _{t \rightarrow \infty} \frac{\log R_{j}(t)}{t}<0 . \\
& \lim _{t \rightarrow \infty} \frac{\log \gamma_{j}(t)}{t}<0 .
\end{aligned}
$$

Theorem 1.1 shows that the correlation function $R_{j}$ decays exponentially if and only if $\gamma_{j}$ decays exponentially.

We show similar facts also for the discrete KMO-Langevin equations. 


\section{§2. Relation between the supports of two measures}

First we recall the notation of [3]:

$$
\begin{gathered}
\Sigma_{2}=\{\sigma ; \sigma \text { is a Borel measure on }[0, \infty) \text { such that } \sigma(\{0\})=0 \\
\left.0<\sigma([0, \infty))<\infty \text { and } \int_{0}^{\infty} \lambda^{-1} \sigma(d \lambda)<\infty\right\}
\end{gathered}
$$

$$
\begin{gathered}
\mathcal{L}_{1}=\{(\alpha, \beta, \rho) ; \alpha>0, \beta>0 \text { and } \rho \text { is a Borel measure on }[0, \infty) \\
\text { such that } \left.\rho(\{0\})=0 \text { and } \int_{0}^{\infty} \frac{1}{1+t} \rho(d t)<\infty\right\}
\end{gathered}
$$

By Theorem 8.5 in [3], the relation

$$
\frac{1}{2 \pi} \int_{0}^{\infty} \frac{1}{\lambda-i \zeta} \sigma(d \lambda)=\frac{\alpha}{\sqrt{2 \pi}}\left(\beta-i \zeta-i \zeta \int_{0}^{\infty} \frac{1}{\lambda-i \zeta} \rho(d \lambda)\right)^{-1}\left(\zeta \in \mathbf{C}^{+}\right)
$$

defines a bijection $\sigma \rightarrow L_{2}(\sigma)=(\alpha, \beta, \rho)$ from $\Sigma_{2}$ onto $\mathcal{L}_{1}$.

In this section, we show a relation between the supports of two measures $\sigma$ and $\rho$ in (2.3). Our principal tool is the approximation method by discrete measures. We define

$$
\begin{aligned}
& \mathcal{L}_{d}=\left\{(\alpha, \beta, \rho) \in \mathcal{L}_{1} ; \rho=\sum_{n=1}^{N} \rho_{n} \delta_{q_{n}} \text { for some } N \in \mathbf{N},\right. \text { where } \\
&\left.\rho_{n}>0(n=1, \ldots, N) \text { and } 0<q_{1}<q_{2}<\cdots<q_{N}<\infty\right\}
\end{aligned}
$$

$$
\begin{aligned}
\Sigma_{d}=\left\{\sigma \in \Sigma_{2} ; \sigma=\right. & \sum_{n=0}^{N} \sigma_{n} \delta_{p_{n}} \text { for some } N \in \mathbf{N}, \text { where } \\
& \left.\sigma_{n}>0(n=0, \ldots, N) \text { and } 0<p_{0}<p_{1}<\cdots<p_{N}<\infty\right\} .
\end{aligned}
$$

Proposition 2.1. (i) $L_{2}\left(\Sigma_{d}\right)=\mathcal{L}_{d}$.

(ii) Let $\sigma \in \Sigma_{d}$ and $(\alpha, \beta, \rho)=L_{2}(\sigma)$. Then we have for some $N \in \mathbf{N}$,

$$
\begin{array}{ll}
\sigma=\sum_{n=0}^{N} \sigma_{n} \delta_{p_{n}}, & \sigma_{n}>0(n=0, \ldots, N), \\
\rho=\sum_{n=1}^{N} \rho_{n} \delta_{q_{n}}, & \rho_{n}>0(n=1, \ldots, N)
\end{array}
$$


with

$$
0<p_{n}<q_{n+1}<p_{n+1} \quad(n=0, \ldots, N-1) .
$$

Proof. (i) First let $\sigma=\sum_{n=0}^{N} \sigma_{n} \delta_{p_{n}} \in \Sigma_{d}$. We put

$$
A(z)=\int_{[0, \infty)} \frac{1}{\lambda-z} \sigma(d \lambda) \quad(\operatorname{Re} z<0) .
$$

Then

$$
A(z)=\sum_{n=0}^{N} \frac{\sigma_{n}}{p_{n}-z}=f(z) / \prod_{n=0}^{N}\left(p_{n}-z\right)
$$

where

$$
f(z)=\sum_{n=0}^{N} \sigma_{n} \prod_{k \neq n}\left(p_{k}-z\right)=(-1)^{N} \sigma([0, \infty)) z^{N}+\cdots .
$$

Since $f\left(p_{n}\right)=\sigma_{n} \prod_{k \neq n}\left(p_{k}-p_{n}\right)$, we have $\operatorname{sgn}\left(f\left(p_{n}\right)\right)=(-1)^{n}(n=0, \ldots, N)$. Therefore, there exist $N$ positive numbers $q_{n}(n=1, \ldots, N)$ such that both (2.8) and the following hold:

$$
f(z)=\sigma([0, \infty)) \prod_{n=1}^{N}\left(q_{n}-z\right)
$$

We put $\alpha=\sigma([0, \infty)) / \sqrt{2 \pi}, \beta=\prod_{n=0}^{N} p_{n} / \prod_{n=1}^{N} q_{n}$ and

$$
\frac{\prod_{n=0}^{N}\left(p_{n}-z\right)}{\prod_{n=1}^{N}\left(q_{n}-z\right)}-(\beta-z)=\frac{h(z)}{\prod_{n=1}^{N}\left(q_{n}-z\right)},
$$

where

$$
h(z)=\prod_{n=0}^{N}\left(p_{n}-z\right)-(\beta-z) \prod_{n=1}^{N}\left(q_{n}-z\right) .
$$

Since $h(0)=0, k(z)=h(z) / z$ is a polynomial of degree $\leq N-1$ and it holds that

$$
\frac{k(z)}{\prod_{n=1}^{N}\left(q_{n}-z\right)}=-\sum_{n=1}^{N} \frac{\rho_{n}}{q_{n}-z}
$$

where

$$
\rho_{n}=-\frac{k\left(q_{n}\right)}{\prod_{k \neq n}\left(q_{k}-q_{n}\right)}=-\frac{\prod_{k=0}^{N}\left(p_{k}-q_{n}\right)}{q_{n} \prod_{k \neq n}\left(q_{k}-q_{n}\right)}>0, \quad(n=1, \ldots, N) .
$$


Thus if we put $\rho=\sum_{n=1}^{N} \rho_{n} \delta_{q_{n}}, \sigma$ and $(\alpha, \beta, \rho)$ satisfy $(2.3)$, so that $L_{2}(\sigma)=$ $(\alpha, \beta, \rho)$ follows. put

(ii) Conversely, let $(\alpha, \beta, \rho) \in \mathcal{L}_{d}$. Then $\rho$ is of the form $\rho=\sum_{n=1}^{N} \rho_{n} \delta_{q_{n}}$. We

$$
B(z)=\alpha \sqrt{2 \pi}\left(\beta-z-z \int_{0}^{\infty} \frac{1}{\lambda-z} \rho(d \lambda)\right)^{-1}
$$

Then we have

$$
B(z)=\alpha \sqrt{2 \pi} \prod_{n=1}^{N}\left(q_{n}-z\right) / g(z)
$$

where

$$
g(z)=(\beta-z) \prod_{n=1}^{N}\left(q_{n}-z\right)-z \sum_{n=1}^{N} \rho_{n} \prod_{k \neq n}\left(q_{k}-z\right)=(-1)^{N+1} z^{N+1}+\cdots
$$

Since $g\left(q_{n}\right)=-q_{n} \rho_{n} \prod_{k \neq n}\left(q_{k}-q_{n}\right)$, it holds that $\operatorname{sgn}\left(g\left(q_{n}\right)\right)=(-1)^{n}(n=$ $1, \ldots, N)$. We also see that $\operatorname{sgn}(g(0))=1$. Therefore, there exist $N+1$ positive numbers $p_{n}(n=0, \ldots, N)$ such that both $(2.8)$ and the following hold:

$$
g(z)=\prod_{n=0}^{N}\left(p_{n}-z\right)
$$

Then we have

$$
B(z)=\frac{\alpha \sqrt{2 \pi} \prod_{n=1}^{N}\left(q_{n}-z\right)}{\prod_{n=0}^{N}\left(p_{n}-z\right)}=\sum_{n=0}^{N} \frac{\sigma_{n}}{p_{n}-z}
$$

where

$$
\sigma_{n}=\alpha \sqrt{2 \pi} \prod_{k=1}^{N}\left(q_{k}-p_{n}\right) / \prod_{k \neq n}\left(p_{k}-p_{n}\right)>0 \quad(n=0, \ldots, N) .
$$

Thus if we put $\sigma=\sum_{n=0}^{N} \sigma_{n} \delta_{p_{n}}$, then (2.3) and therefore $\sigma=L_{2}^{-1}((\alpha, \beta, \rho))$ hold. This completes the proof of the proposition.

Let $I$ be an interval of $\mathbf{R}$. Let $\sigma$ be a bounded Borel measure on $I$. We denote by $\lambda_{0}(\sigma)$ and $\lambda_{\infty}(\sigma)$ the infimum and the supremum of $\operatorname{supp} \sigma$, respectively:

$$
\lambda_{0}(\sigma)=\inf \{\lambda \in I ; \lambda \in \operatorname{supp} \sigma\}, \quad \lambda_{\infty}(\sigma)=\sup \{\lambda \in I ; \lambda \in \operatorname{supp} \sigma\} .
$$

Theorem 2.2. Let $\sigma \in \Sigma_{2}$ and $(\alpha, \beta, \rho)=L_{2}(\sigma)$. Then

$$
\lambda_{0}(\sigma) \leq \lambda_{0}(\rho) \leq \lambda_{\infty}(\rho) \leq \lambda_{\infty}(\sigma)
$$


Proof. The proof of the third inequality is almost the same as the first inequality, so that we prove only the first one. We may assume $\lambda_{0}(\sigma)>0$. We choose $\sigma^{(n)} \in \Sigma_{d}(n=1,2, \ldots)$ so that $\operatorname{supp} \sigma^{(n)} \subset\left[\lambda_{0}(\sigma), \infty\right)$ and $\mathrm{w}-\lim _{n \rightarrow \infty} \sigma^{(n)}=\sigma$ on $\left[\lambda_{0}(\sigma), \infty\right]$. Here we regard $\sigma^{(n)}$ and $\sigma$ as bounded Borel measures on $\left[\lambda_{0}(\sigma), \infty\right]$ by $\sigma^{(n)}(\infty)=\sigma(\infty)=0$. If we put $L_{2}\left(\sigma^{(n)}\right)=\left(\alpha^{(n)}, \beta^{(n)}, \rho^{(n)}\right)$, then it holds that

$$
\int_{\left[\lambda_{0}(\sigma), \infty\right)} \frac{1}{\lambda+1} \rho^{(n)}(d \lambda)=\frac{\alpha^{(n)} \sqrt{2 \pi}}{\int_{\left[\lambda_{0}(\sigma), \infty\right)}(\lambda+1)^{-1} \sigma^{(n)}(d \lambda)}-\beta^{(n)}-1 .
$$

In view of Theorem 8.2 in [3], we have

$$
\lim _{n \rightarrow \infty} \alpha^{(n)}=\frac{\sigma\left(\left[\lambda_{0}(\sigma), \infty\right)\right)}{\sqrt{2 \pi}}=\alpha, \quad \lim _{n \rightarrow \infty} \beta^{(n)}=\frac{\sigma\left(\left[\lambda_{0}(\sigma), \infty\right)\right)}{\int_{\left[\lambda_{0}(\sigma), \infty\right)} \lambda^{-1} \sigma(d \lambda)}=\beta
$$

Furthermore, we have

$$
\lim _{n \rightarrow \infty} \int_{\left[\lambda_{0}(\sigma), \infty\right)} \frac{1}{\lambda+1} \sigma^{(n)}(d \lambda)=\int_{\left[\lambda_{0}(\sigma), \infty\right)} \frac{1}{\lambda+1} \sigma(d \lambda) .
$$

Then if we put $\widetilde{\rho}^{(n)}=(\lambda+1)^{-1} \rho^{(n)}$, it holds that $\sup _{n} \widetilde{\rho}^{(n)}\left(\left[\lambda_{0}(\sigma), \infty\right]\right)<\infty$. Here we put $\widetilde{\rho}^{(n)}(\{\infty\})=0$. Therefore there exist a subsequence $n_{k}(k=1,2, \ldots)$ and a bounded Borel measure $\widetilde{\rho}$ on $\left[\lambda_{0}(\sigma), \infty\right]$ such that

$$
\mathrm{W}-\lim _{k \rightarrow \infty} \widetilde{\rho}^{\left(n_{k}\right)}=\tilde{\rho} \quad \text { on }\left[\lambda_{0}(\sigma), \infty\right] .
$$

We put $\rho^{\prime}=(\lambda+1) \tilde{\rho}$. Then using this subsequence, we obtain for any $\zeta \in \mathbf{C}^{+}$,

$$
\begin{aligned}
& \frac{1}{2 \pi} \int_{\left[\lambda_{0}(\sigma), \infty\right)} \frac{1}{\lambda-i \zeta} \sigma(d \lambda) \\
& \quad=\frac{\alpha}{\sqrt{2 \pi} c}\left(\frac{\beta}{c}-i \zeta-i \zeta \int_{\left[\lambda_{0}(\sigma), \infty\right)} \frac{1}{\lambda-i \zeta} \frac{\rho^{\prime}}{c}(d \lambda)\right)^{-1}
\end{aligned}
$$

where $c=\widetilde{\rho}(\{\infty\})+1$. Then it follows from the uniqueness that $c=1$ and $\rho=\rho^{\prime}$, so that $\lambda_{0}(\sigma) \leq \lambda_{0}(\rho)$. This completes the proof of the theorem.

TheOREM 2.3. Let $\sigma \in \Sigma_{2}$ and $L_{2}(\sigma)=(\alpha, \beta, \rho)$.

(i) If $\lambda_{0}(\rho)>0$, then for some $\sigma_{0} \geq 0, p_{0} \in\left(0, \lambda_{0}(\rho)\right)$, and $\mu_{1} \in \Sigma_{2}$ with $\operatorname{supp} \mu_{1} \subset\left[\lambda_{0}(\rho), \infty\right)$, it holds that $\sigma=\sigma_{0} \delta_{p_{0}}+\mu_{1}$.

(ii) If $\lambda_{\infty}(\rho)<\infty$, then for some $\sigma_{\infty} \geq 0, p_{\infty} \in\left(\lambda_{\infty}(\rho), \infty\right)$, and $\mu_{2} \in \Sigma_{2}$ with $\operatorname{supp} \mu_{2} \subset\left[0, \lambda_{\infty}(\rho)\right]$ it holds that $\sigma=\mu_{2}+\sigma_{\infty} \delta_{p_{\infty}}$. 
Proof. The proof of (ii) is almost the same as (i), so that we prove only (i). We put $\widetilde{\rho}=(\lambda+1)^{-1} \rho$. We regard $\rho$ as a bounded Borel measure on $\left[\lambda_{0}(\rho), \infty\right]$ by $\widetilde{\rho}(\{\infty\})=0$. We choose $\widetilde{\rho}^{(n)}(n=1,2, \ldots)$ so that $\left(\alpha, \beta, \widetilde{\rho}^{(n)}\right) \in \mathcal{L}_{d}, \operatorname{supp} \widetilde{\rho}^{(n)} \subset$ $\left[\lambda_{0}(\rho), \infty\right)$ and $\mathrm{w}-\lim _{n \rightarrow \infty} \widetilde{\rho}^{(n)}=\tilde{\rho}$ on $\left[\lambda_{0}(\rho), \infty\right]$. By considering $\widetilde{\rho}^{(n)}+n^{-1} \delta_{\lambda_{0}(\rho)}$ instead of $\widetilde{\rho}^{(n)}$ if necessary, we may assume that $\lambda_{0}(\rho) \in \operatorname{supp}\left(\widetilde{\rho}^{(n)}\right)(n=1,2, \ldots)$. We put $\rho^{(n)}=(\lambda+1) \widetilde{\rho}^{(n)}$. Then $\rho^{(n)}$ is of the form

$$
\rho^{(n)}=\sum_{k=1}^{N^{(n)}} \rho_{k}^{(n)} \delta_{q_{k}^{(n)}}
$$

with

$$
\lambda_{0}(\rho)=q_{1}^{(n)}<q_{2}^{(n)}<\cdots<q_{N^{(n)}}^{(n)}, \quad \rho_{k}^{(n)}>0\left(k=1,2, \ldots, N^{(n)}\right) .
$$

We put $\sigma^{(n)}=L_{2}^{-1}\left(\left(\alpha, \beta, \rho^{(n)}\right)\right)$. By Proposition 2.1 , we see that

$$
\sigma^{(n)}=\sigma_{0}^{(n)} \delta_{p_{0}^{(n)}}+\mu_{1}^{(n)}
$$

where $\sigma_{0}^{(n)}>0,0<p_{0}^{(n)}<\lambda_{0}(\rho)$ and $\mu_{1}^{(n)} \in \Sigma_{d}$ with supp $\mu_{1}^{(n)} \subset\left[\lambda_{0}(\rho), \infty\right)$. It follows from Theorem 8.2 in [3] that $\sigma^{(n)}([0, \infty))=\sqrt{2 \pi} \alpha$, and so there exist a subsequence $n_{k}(k=1,2, \ldots)$, constants $\sigma_{0} \geq 0, p_{0} \in\left[0, \lambda_{0}(\rho)\right]$ and a bounded Borel measure $\mu_{1}^{\prime} \in \Sigma_{2}$ with supp $\mu_{1}^{\prime} \subset\left[\lambda_{0}(\rho), \infty\right]$ such that

$$
\lim _{k \rightarrow \infty} \sigma_{0}^{\left(n_{k}\right)}=\sigma_{0}, \quad \lim _{k \rightarrow \infty} p_{0}^{\left(n_{k}\right)}=p_{0}, \quad \text { w- } \lim _{k \rightarrow \infty} \mu_{1}^{\left(n_{k}\right)}=\mu_{1}^{\prime} \quad \text { on }\left[\lambda_{0}(\rho), \infty\right] .
$$

We define $\mu_{1} \in \Sigma_{2}$ by $\mu_{1}(d \lambda)=\mu_{1}^{\prime}\left(d \lambda \cap\left[\lambda_{0}(\rho), \infty\right)\right)$. Then letting $k \rightarrow \infty$, we obtain

$$
\begin{aligned}
& \frac{\sigma_{0}}{2 \pi\left(p_{0}-i \zeta\right)}+\frac{1}{2 \pi} \int_{\left[\lambda_{0}(\rho), \infty\right)} \frac{1}{\lambda-i \zeta} \mu_{1}(d \lambda) \\
& \quad=\frac{\alpha}{\sqrt{2 \pi}}\left(\beta-i \zeta-i \zeta \int_{\left[\lambda_{0}(\rho), \infty\right)} \frac{\lambda+1}{\lambda-i \zeta} \widetilde{\rho}(d \lambda)\right)^{-1} \quad\left(\zeta \in \mathbf{C}^{+}\right) .
\end{aligned}
$$

If $p_{0}=0$, we obtain, by substituting $i y$ into $\zeta$ in $(2.3)$,

$$
\frac{\sigma_{0}}{y} \leq \frac{\alpha \sqrt{2 \pi}}{\beta} \quad(y>0)
$$

which shows $\sigma_{0}=0$. Thus we see that $\sigma=\sigma_{0} \delta_{p_{0}}+\mu_{1}$ and this completes the proof. 


\section{§3. Characterization of the exponential decay (1)}

In this section, we consider the continuous KMO-Langevin equations and prove Theorem 1.1.

First we recall additional notations in [3]. Let

$$
\begin{gathered}
\Sigma_{1}=\{\sigma ; \sigma \text { is a Borel measure on }[0, \infty) \text { such that } \sigma(\{0\})=0, \\
\left.0<\sigma([0, \infty))<\infty \text { and } \int_{0}^{\infty}\left(\lambda+\lambda^{-1}\right) \sigma(d \lambda)<\infty\right\}
\end{gathered}
$$

Then by Theorems 2.1, 3.1, Lemma 2.6 and (2.19) in [3], the relation

$$
\sigma(d \lambda)=\frac{1}{2 \pi}\left(\int_{0}^{\infty} \frac{1}{\lambda+\lambda^{\prime}} \nu\left(d \lambda^{\prime}\right)\right) \nu(d \lambda), \quad L_{2}(\nu)=(\alpha, \beta, \rho)
$$

defines a bijection $\sigma \rightarrow L_{1}(\sigma)=(\alpha, \beta, \rho)$ from $\Sigma_{1}$ onto $\mathcal{L}_{1}$.

Theorem 3.1. Let $j=1$ or 2 . Let $\sigma_{j} \in \Sigma_{j}$ and $L_{j}\left(\sigma_{j}\right)=\left(\alpha_{j}, \beta_{j}, \rho_{j}\right)$. Then

(i) $\lambda_{0}\left(\sigma_{j}\right)>0$ if and only if $\lambda_{0}\left(\rho_{j}\right)>0$.

(ii) $\lambda_{\infty}\left(\sigma_{j}\right)<\infty$ if and only if $\lambda_{\infty}\left(\rho_{j}\right)<\infty$.

Proof. The case of $j=2$ follows immediately from Theorems 2.2 and 2.3. Next we consider the case of $j=1$. We put $\nu=L_{2}{ }^{-1}\left(\left(\alpha_{1}, \beta_{1}, \rho_{1}\right)\right)$. Then it follows from the case of $j=2$ that

$$
\lambda_{0}(\nu)>0 \Longleftrightarrow \lambda_{0}\left(\rho_{1}\right)>0 \text { and } \lambda_{\infty}(\nu)<\infty \Longleftrightarrow \lambda_{\infty}\left(\rho_{1}\right)<\infty .
$$

On the other hand, by (3.2), we see that $\operatorname{supp} \sigma_{1}=\operatorname{supp} \nu$. Thus the case of $j=1$ follows and this completes the proof.

Proposition 3.2. Let $\sigma$ be a bounded Borel measure on $[0, \infty)$. We put for $t \in \mathbf{R}, R(t)=\int_{0}^{\infty} e^{-t \lambda} \sigma(d \lambda)$. Then we have

$$
\lim _{t \rightarrow \infty} \frac{\log R(t)}{t}=-\lambda_{0}(\sigma)
$$

Proof. For any $\varepsilon>0$, take positive numbers $a, b$ such that $\lambda_{0}(\sigma) \leq a<b<$ $\lambda_{0}(\sigma)+\varepsilon$ and $\sigma([a, b])>0$. Since

$$
e^{-t\left(\lambda_{0}(\sigma)+\varepsilon\right)} \sigma([a, b]) \leq R(t)=\int_{\lambda_{0}(\sigma)}^{\infty} e^{-t \lambda} \sigma(d \lambda) \leq e^{-t \lambda_{0}(\sigma)} \sigma\left(\left[\lambda_{0}(\sigma), \infty\right)\right),
$$

we have

$$
-\left(\lambda_{0}(\sigma)+\varepsilon\right) \leq \liminf _{t \rightarrow \infty} \frac{\log R(t)}{t} \leq \limsup _{t \rightarrow \infty} \frac{\log R(t)}{t} \leq-\lambda_{0}(\sigma) .
$$


Since $\varepsilon$ is arbirtrary, we obtain (3.1).

Proof of Theorem 1.1. In view of Proposition 3.2, it is enough to show that $\lambda_{0}\left(\sigma_{j}\right)>0 \Longleftrightarrow \lambda_{0}\left(\rho_{j}\right)>0$ but this is a straight consequence of Theorem 3.1. Thus the proof of the theorem is complete.

\section{§4. Characterization of the exponential decay (2)}

In this section, we show a result which is similar to Theorem 1.1 for the discrete KMO-Langevin equations.

We define

$$
\begin{aligned}
& \Sigma_{1}^{d}=\{\sigma ; \sigma \text { is a bounded Borel measure on }[-1,1] \text { such that } \\
& \left.\qquad(\{-1,1\})=0 \text { and } \int_{-1}^{1}\left(\frac{1}{1+t}+\frac{1}{1-t}\right) \sigma(d t)<\infty\right\},
\end{aligned}
$$

$$
\mathcal{L}_{1}^{d}=\{(\alpha, \beta, \rho) ; \alpha>0, \beta>0 \text { and } \rho \text { is a bounded }
$$

Borel measure on $[-1,1]$ such that $\rho(\{-1,1\})=0\}$.

By Theorem 3.1 in [6], the relation

$$
\begin{aligned}
& \frac{1}{2 \pi} \int_{-1}^{1} \frac{1}{1-t z} \sigma(d t) \\
& =\frac{\alpha}{\sqrt{2 \pi}}\left(\beta(1+z)+1-z+\left(1-z^{2}\right) \int_{-1}^{1} \frac{1}{1-t z} \rho(d t)\right)^{-1} \quad(|z|<1)
\end{aligned}
$$

defines a bijection $\sigma \rightarrow L_{2}(\sigma)=(\alpha, \beta, \rho)$ from $\Sigma_{1}^{d}$ onto $\mathcal{L}_{1}^{d}$. Furthermore, by Theorems $4.1,4.2$ and 4.4 in [5], we see that the relation

$$
\sigma(d t)=\frac{1}{2 \pi}\left(\int_{-1}^{1} \frac{1}{1-t s} \nu(d s)\right) \nu(d t), \quad L_{2}(\nu)=(\alpha, \beta, \rho)
$$

defines another bijection $\sigma \rightarrow L_{1}(\sigma)=(\alpha, \beta, \rho)$ from $\Sigma_{1}^{d}$ onto $\mathcal{L}_{1}^{d}$.

Now we consider the first discrete KMO-Langevin equation

$$
\begin{aligned}
& X(n)-X(n-1) \\
& \quad=-\beta_{1}(X(n)+X(n-1))-\left(\gamma_{1} * X\right)(n)+\alpha_{1} \xi(n) \quad \text { a.s. }(n \in \mathbf{Z})
\end{aligned}
$$

and the second discrete KMO-Langevin equation

$$
\begin{aligned}
& X(n)-X(n-1) \\
& \quad=-\beta_{2}(X(n)+X(n-1))-\left(\gamma_{2} * X\right)(n)+\alpha_{2} I(n) \quad \text { a.s. }(n \in \mathbf{Z}) .
\end{aligned}
$$


Here for $j=1,2$,

$$
\gamma_{j}(n)=\left\{\begin{array}{l}
0 \quad(n=-1,-2, \ldots) \\
\int_{-1}^{1} t^{n} \rho_{j}(d t) \quad(n=0,1) \\
\int_{-1}^{1} t^{n-2}\left(t^{2}-1\right) \rho_{j}(d t) \quad(n=2,3, \ldots)
\end{array}\right.
$$

with $\left(\alpha_{j}, \beta_{j}, \rho_{j}\right) \in \mathcal{L}_{1}^{d} . \Xi=(\xi(n) ; n \in \mathbf{Z})$ in (4.5) is a normalized Gaussian white noise or a sequence of independent Gaussian random variables with mean 0 and variance $1 . \mathbf{I}=(I(n) ; n \in \mathbf{Z})$ in (4.6) is a real stationary Gaussian process named the Kubo noise associated with the solution $\mathbf{X}=(X(n) ; n \in \mathbf{Z})$ of (4.6) (see [6]).

The equation (4.5) (resp. (4.6)) has a unique real stationary solution $\mathbf{X}_{1}=$ $\left(X_{1}(n) ; n \in \mathbf{Z}\right)\left(\right.$ resp. $\left.\mathbf{X}_{2}=\left(X_{2}(n) ; n \in \mathbf{Z}\right)\right)$ with mean 0 and covariance $R_{1}$ (resp. $R_{2}$ ) of the form

$$
R_{j}(n)=\int_{-1}^{1} t^{|n|} \sigma_{j}(d t) \quad(n \in \mathbf{Z})
$$

where $\sigma_{j}=L_{j}^{-1}\left(\left(\alpha_{j}, \beta_{j}, \rho_{j}\right)\right) \in \Sigma_{1}^{d}(j=1,2)$. The property (4.8) is called the reflection positivity.

In this section, we use the following notation: a real sequence $(a(n))_{n=0}^{\infty}$ is said to be of exponential decay if there exist two constants $c>0$ and $p \in(0,1)$ such that

$$
|a(n)| \leq c p^{n} \quad(n=0,1, \ldots) .
$$

In the same way as Proposition 2.1, we obtain

Proposition 4.1. Let $\sigma$ be a bounded Borel measure on $[-1,1]$ such that $\sigma(\{-1,1\})=0$. We put $R(n)=\int_{-1}^{1} t^{n} \sigma(d t)(n=0,1, \ldots)$. Then $(R(n))_{n=0}^{\infty}$ is of exponential decay if and only if

$$
-1<\lambda_{0}(\sigma) \text { and } \quad \lambda_{\infty}(\sigma)<1
$$

The following theorem is a discrete analogue to Theorem 1.1.

THEOREM 4.2. Let $j=1$ or 2 . Then the following (i) and (ii) is equivalent:

(i) $\left(\gamma_{j}(n)\right)_{n=0}^{\infty}$ is of exponential decay.

(ii) $\left(R_{j}(n)\right)_{n=0}^{\infty}$ is of exponential decay.

Proof. Let $\sigma_{j} \in \Sigma_{1}^{d}$ and $\left(\alpha_{j}, \beta_{j}, \rho_{j}\right)=L_{j}\left(\sigma_{j}\right)$. In view of Proposition 4.1, it is enough to show that

$$
\lambda_{0}\left(\sigma_{j}\right)>-1, \quad \lambda_{\infty}\left(\sigma_{j}\right)<1 \Longleftrightarrow \lambda_{0}\left(\rho_{j}\right)>-1, \quad \lambda_{\infty}\left(\rho_{j}\right)<1 .
$$


When $j=1$, we use a homeomorphism $\varphi$ from $(-1,1]$ to $[0, \infty)$ :

$$
\varphi(t)=\frac{1-t}{1+t}
$$

to get a bounded Borel measure $\sigma_{c}$ on $[0, \infty)$ by $\sigma_{c}=\varphi(\sigma)$. We also define a new triple $\left(\alpha_{c}, \beta_{c}, \rho_{c}\right)$ by

$$
\alpha_{c}=\frac{\alpha_{1}}{\sqrt{2}}, \quad \beta_{c}=\beta_{1} \quad \text { and } \quad \rho_{c}=\varphi\left(\frac{1}{1+\cdot} \rho_{1}\right) .
$$

By the proofs of Theorems 4.1 and 5.1 in [5], we see that $\sigma_{c}=L_{1}\left(\sigma_{c}, \beta_{c}, \rho_{c}\right)$. Therefore by Theorem 3.1, it holds that

$$
\lambda_{0}\left(\sigma_{c}\right)>0, \quad \lambda_{\infty}\left(\sigma_{c}\right)<\infty
$$

is equivalent to

$$
\lambda_{0}\left(\rho_{c}\right)>0, \quad \lambda_{\infty}\left(\rho_{c}\right)<\infty .
$$

On the other hand, (4.10) and (4.11) are equivalent to

$$
\lambda_{0}\left(\sigma_{j}\right)>-1, \quad \lambda_{\infty}\left(\sigma_{j}\right)<1
$$

and

$$
\lambda_{0}\left(\rho_{j}\right)>-1, \quad \lambda_{\infty}\left(\rho_{j}\right)<1,
$$

respectively. Thus the case of $j=1$ is proved.

Next we consider the case of $j=2$. We put $\nu=L_{1}^{-1}\left(\left(\alpha_{2}, \beta_{2}, \rho_{2}\right)\right)$. Then it follows from the case of $j=1$ that

$$
\lambda_{0}(\nu)>-1, \quad \lambda_{\infty}(\nu)<1 \Longleftrightarrow \lambda_{0}\left(\rho_{2}\right)>-1, \quad \lambda_{\infty}\left(\rho_{2}\right)<1 .
$$

On the other hand, by (4.4), we see that $\operatorname{supp}\left(\sigma_{2}\right)=\operatorname{supp}(\nu)$. Thus the case of $j=2$ is proved and this completes the proof of the theorem.

\section{References}

[1] A. Inoue, The Alder-Wainwright effect for stationary processes with reflection positivity, J. Math. Soc. Japan, 43 (1991), 515-526.

[ 2 ] A. Inoue, The Alder-Wainwright effect for stationary processes with reflection positivity (II), Osaka J. Math., 28 (1991), 537-561.

[3] Y. Okabe, On KMO-Langevin equations for stationary Gaussian processes with Tpositivity, J. Fac. Sci. Univ. Tokyo Sect. IA, 33 (1986), 1-56.

[4] Y. Okabe, On the theory of the Brownian motion with the Alder-Wainwright effect, J. Statist. Phys., 45 (1986), 953-981. 
[ 5 ] Y. Okabe, On the theory of disrete KMO-Langevin equations with reflection positivity (I), Hokkaido Math. J., 16 (1987), 315-341.

[ 6 ] Y. Okabe, On the theory of disrete KMO-Langevin equations with reflection positivity (II), Hokkaido Math. J., 17 (1988), 1-44.

[ 7 ] Y. Okabe, On long time tails of correlation functions for KMO-Langevin equations, Probability Theory and Mathematical Statistics (S. Watanabe and Yu. V. Prokhorov, ed.), Lecture Notes in Math., 1299, Springer, 1988, pp. 391-397.

\section{Department of MATHEMatics \\ FACUlTy OF SCIENCE \\ HOKKAIDO UNIVERSITY \\ SAPPORO 060, JAPAN}

